\title{
Catalogando Requisitos Não-Funcionais de Sistemas Embarcados
}

\author{
Reinaldo Silva $^{1,2}$, Jaelson Castro ${ }^{2}$, João Pimentel ${ }^{3}$ \\ ${ }^{1}$ Instituto Federal de Maranhão, Brasil \\ reinaldo.silvalifma.edu.br \\ ${ }^{2}$ Universidade Federal de Pernambuco, Brasil \\ $\{$ ras $12, j b c\} @ c i n . u f p e . b r$ \\ ${ }^{3}$ Universidade Federal Rural de Pernambuco, Brasil \\ joao.hcpimentel@ufrpe.br
}

\begin{abstract}
Difficulties in the process of developing embedded systems, such as the treatment of Non-Functional Requirements (RNFs), led developers to seek in requirements engineering concepts and applications to support their construction. Thus, this article addresses the complexity in the treatment of RNFs in embedded systems projects through a catalog of Non-Functional Requirements for embedded systems (NFR4ES). This catalog has the knowledge acquired from the literature and from experts in the field, which was organized for the elicitation and specification of non-functional requirements for embedded systems and can be used for reuse. This catalog was evaluated by a proof of concept and a survey with specialists, in which it was identified as useful and suitable for the elicitation and specification of RNFs in embedded systems.
\end{abstract}

Resumo. As dificuldades no processo de desenvolvimento de sistemas embarcados, como o tratamento de Requisitos Não-Funcionais(RNFs), levaram os desenvolvedores a buscarem na engenharia de requisitos conceitos $e$ aplicações que apoiassem a sua construção. Assim, este artigo aborda a complexidade no tratamento de RNFs em projetos de sistemas embarcados através de um catálogo de Requisitos Não-Funcionais para sistemas embarcados (NFR4ES). Este catálogo possui o conhecimento adquirido a partir da literatura e de especialistas do domínio, que foi organizado para a elicitação e especificação de requisitos não-funcionais para sistemas embarcados e pode ser utilizado para reúso. Este catálogo foi avaliado por uma prova de conceito e uma pesquisa com especialistas, no qual foi apontado como útil e adequado para a elicitação e especificação de RNFs em sistemas embarcados.

\section{Introdução}

No processo de desenvolvimento de um sistema embarcado várias questões devem ser levadas em consideração no que diz respeito ao tratamento de Requisitos NãoFuncionais, tais como: baixo consumo de potência sem comprometimento do desempenho do sistema, questões relacionadas a memória, peso, segurança, tempo-real, 
alta confiabilidade, disponibilidade, entre outros fatores [1].

Requisitos Não-Funcionais (RNFs) são constantemente tratados de forma inadequada em projetos de sistemas embarcados [2]. Estes requisitos, são fundamentais para que o projeto do sistema embarcado seja bem sucedido. Se os RNFsnão forem elicitados de forma correta, podem ocorrer diversos problemas que resultam em má qualidade do sistema, insatisfação dos stakeholders e gastos com a correção do sistema [3]. Em determinados tipos de sistemas, tais como sistemas embarcados e sistemas críticos de segurança (safety critical systems), os RNFs podem ser mais críticos do que os requisitos funcionais, sendo considerados um fator determinante para o sucesso ou falha do sistema [4]. Não obstante, foi observado que a elicitação e a especificação dos RNFs é um grande desafio no desenvolvimento de sistemas embarcados $[2,5]$.

O objetivo geral da pesquisa foi construir um catálogo de RNFs para o domínio de sistemas embarcados, de forma a apoiar as etapas de elicitação e especificação de requisitos [6]. O catálogo foi chamado de NFR4ES (Non-Functional Requirements for Em- bedded Systems). Ele não pretende englobar todos os RNFs presentes nos sistemas embarcados. Em vez disso, inclui um sub-conjunto de RNFs que são relevantes para sistemas embarcados conforme evidências comprobatórias coletadas na literatura especializada.

Este trabalho foi guiado pelas seguintes questões de pesquisa: (i) Quais os principais RNFs que devem ser levados em consideração em projetos de sistemas embarcados?;

(ii) Quais são os inter-relacionamentos entre os principais RNFs para sistemas embarcados?

Para a documentação do catálogo foi adotado o NFR Framework, uma abordagem que tem sido utilizada com sucesso para a representação e análise de RNFs [3]. Seu objetivo é ajudar desenvolvedores na implementação de soluções personalizadas, levando em consideração as características do domínio e do sistema em questão [3]. Nesse fra-mework, os RNFs são representados através de um grafo de interdependência de softgoals conhecido como Softgoal Interdependency Graph (SIG). O catálogo foi avaliado através de uma prova de conceito e pela opinião de especialistas.

Este artigo está estruturado da seguinte forma. A Seção 2 descreve a metodologia para construção do catálogo. Na Seção 3, o catálogo propriamente dito é apresentado. A Seção 4 descreve uma prova de conceito, enquanto a Seção 5 discute uma avaliação por especialistas. Os trabalhos relacionados são discutidos na Seção 6. Por último, o artigo é concluído com a Seção 7. Ele é uma versão estendida do artigo disponível em [7].

\section{Metodologia de Construção do Catálogo}

O processo de construção do catálogo NFR4ES (Non-Functional Requirements for Embedded Systems) é apresentado na Figura 1 através da notação Business Process Model and Notation (BPMN) desenvolvida pela OMG [8].

A primeira atividade do processo foi a identificação de candidatos a RNFs relevantes para sistemas embarcados. A entrada para esta atividade foi um conjunto de 84 artigos que haviam sido identificados durante uma atualização da Revisão Sistemática da Literatura (RSL) sobre engenharia de requisitos para sistemas embarcados [9]. Destes, apenas 45 artigos relatavam RNFs de sistemas embarcados. A partir dos quais foi identificado um conjunto inicial de 52 RNFs. Em seguida, foi realizada uma triagem 
inicial para a eliminação de requisitos duplicados (termos diferentes com o significado equivalente). Essa triagem inicial resultou na exclusão de 15 requisitos.

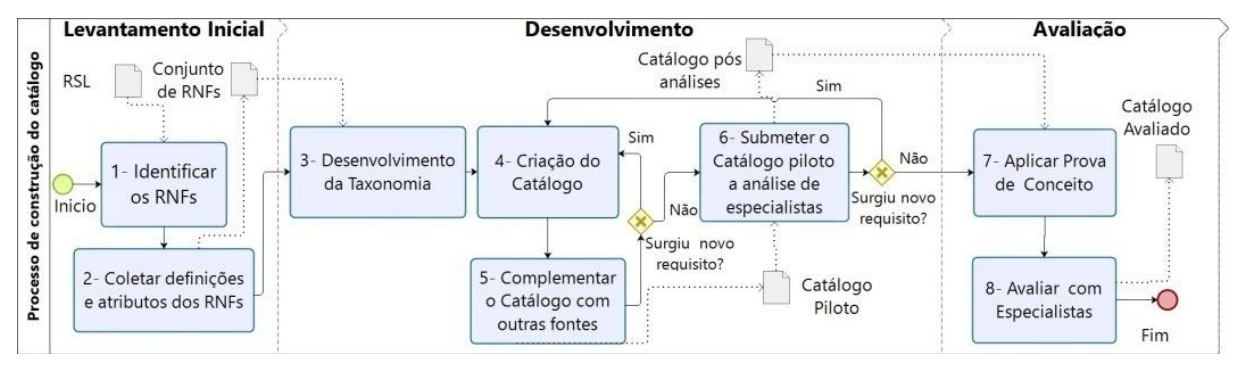

Figura 1. Fases e atividades do processo de construção do catálogo

$\mathrm{Na}$ tarefa 2, buscou-se "Coletar definições dos RNFs" dos 37 requisitos restantes. Ao realizar esta atividade não foi possível coletarmos todas as definições a partir dos 45 artigos originais, pois alguns artigos apenas os citavam superficialmente. Desta forma, foi necessário a busca dessas definições em outras fontes de informação, a saber: artigos acadêmicos, livros acadêmicos, trabalhos de pós-graduação (dissertações e teses), documentos de requisitos de sistemas embarcados, e padrões da indústria.

Mesmo com essa ampla busca, não foi possível encontrar exemplos nem definições de 16 desses requisitos, que estão disponíveis em [6]. Por falta de evidências corroborativas, esses RNFs também foram excluídos do catálogo. Isso não significa que esses RNFs excluídos não poderiam ser pertinentes para alguns projetos de sistemas embarcados, mas em trabalhos futuros seria interessante investigar a relevância desses RNFs em outros projetos.

Como resultado da tarefa 2, foi construído um conjunto inicial de 21 RNFs com definições. Estes foram: área, disponibilidade, custo, consumo de energia, manutenibilidade, desempenho, portabilidade, privacidade, confiabilidade, taxa de repetição, disponibilidade de recursos, consumo de recursos, tempo de resposta, segurança (safety), segurança (security), throughput, timeout, tempo, delay, usabilidade e taxa de utilização.

Na fase de Desenvolvimento do catálogo, realizamos a construção de uma taxonomia de RNFs para sistemas embarcados (tarefa 3) e a construção do Catálogo NFR4ES (tarefa 4). Além disso, melhoramos e complementamos o nosso catálogo a partir de outras fontes de informação como livros técnicos e artigos científicos que não foram identificados na RSL (tarefa 5). No final desta fase, uma versão piloto do catálogo foi gerada para ser submetida a uma análise preliminar por especialistas (tarefa 6).

Na tarefa "Desenvolvimento da Taxononomia" foi criada uma árvore taxonômica com os 21 RNFs obtidos na fase anterior. O objetivo da construção dessa taxonomia foi auxiliar na compreensão desses requisitos e iniciar a sua estruturação. Este foi um passo intermediário para facilitar a criação dos Softgoal Interdependency Graphs (SIGs) utilizados no catálogo.

Após a construção da Taxonomia, foi iniciada a criação do nosso Catálogo de RNFs, organizando todos os RNFs encontrados nas etapas anteriores com suas definições e seus exemplos (tarefa 4). Foi realizada a construção de um grafo Softgoal Interdependency Graph (SIG) com os RNFs, adotando a notação do NFR Framework proposto por [3]. Além disso, nesta tarefa foi adotado o cartão de especificação baseado no cartão 
(snowcard) do processo Volere [9] para a escrita dos exemplos.

$\mathrm{Na}$ tarefa 5 (“Complementar o catálogo com outras fontes"), complementamos o catálogo com buscas ad hoc na literatura acadêmica, incluindo artigos acadêmicos, livros acadêmicos, dissertações e teses.

Na tarefa 6 ("Submeter o Catálogo piloto a análise de especialistas"), versões preliminares do catálogo foram submetidas à análise de quatro especialistas do domínio, visando a verificação da consistência dos RNFs em aspectos como: relevância, corretude e aplicabilidade em cenários reais na indústria. Os perfis dos especialistas que realizaram a análise do catálogo foram os seguintes: dois profissionais da acadêmia com Doutorado que também atuam na indústria e possuem experiência em projetos de sistemas embarcados; e dois desenvolvedores experientes que possuem mestrado na área de sistemas embarcados e atualmente trabalham na indústria.

A análise feita pelos especialistas resultou no aperfeiçoamento do catálogo através de sugestões de novos RNFs, ajustes nas definições e ajustes na hierarquia dos requisitos. Adicionalmente, um dos papéis desses especialistas foi na identificação das correlações entres os RNFs apresentados no catálogo - isso é, da influência que um RNF exerce em outro RNF. Isso foi feito através de uma análise par a par entre os requisitos raiz.

Ao todo, considerando-se múltiplas iterações das tarefas 5 e 6, vinte e três novos RNFs foram identificados durante a fase de desenvolvimento do catálogo: tolerância a falhas, dimensões, peso, formato, durabilidade, facilidade de transporte, não-inflamável, temperatura, impermeabilidade, compatibilidade eletromagnética, prevenção de perigos, registro de eventos, identificação do usuário, acesso seguro à rede, comunicação segura, armazenamento seguro, segurança de conteúdo, resistência a violação, capacidade de aprendizado, proteção contra erros do usuário, adaptabilidade, satisfação do usuário e intuitividade. Consequentemente, o catálogo final (apresentado na Seção 3) contempla 44 RNFs, sendo 21 oriundos da RSL e 23 identificados durante a fase de desenvolvimento do catálogo.

Durante a fase de Avaliação, uma prova de conceito foi executada (tarefa 7) aplicando o catálogo para a elicitação e especificação a posteriori de um sistema embarcado de uma empresa brasileira que atua na área de diagnóstico automatizado de doenças. Esta aplicação foi realizada por um dos autores com base nas informações fornecidas pelo Engenheiro de Hardware responsável pelo projeto na empresa. Posteriormente, uma instanciação do catálogo foi gerada por um dos autores e validada pelo representante da empresa. Maiores detalhes sobre essa prova de conceito são apresentados na Seção 4.

Por fim (tarefa 8), foi realizada uma avaliação através de questionário com dez profissionais que atuam no dom'inio de sistemas embarcados. Nenhum desses profissionais havia participado da fase de Desenvolvimento do catálogo. Os resultados dessa avaliação são descritos na Seção 5.

\section{O Catálogo NFR4ES}

O catálogo final compreende: um Softgoal Interdependency Graph (SIG), mostrando a hierarquia dos RNFs e suas correlações; as definições de cada RNF; e um exemplo para cada requisito folha (isto é, requisitos que não são refinados em outros requisitos). A Figura 2 apresenta uma visão parcial do SIG, sem as correlações. São dez requisitos

raiz, potencialmente refinados em outros requisitos: Confiabilidade, Desempenho, Portabilidade, Manutenibilidade, Estrutura física, Consumo de Energia, Segurança (Safety), 
Custo, Segurança (Security) e Usabilidade. Os RNFs Custo, Consumo de Energia, Manutenibilidade e Portabilidade não apresentam refinamentos pois não foram encontrados detalhamentos sobre os mesmos na literatura de sistemas embarcados. As evidências da aplicabilidade de todos os RNFs do SIG ao domínio de sistemas embarcados estão listadas no Apêndice A.

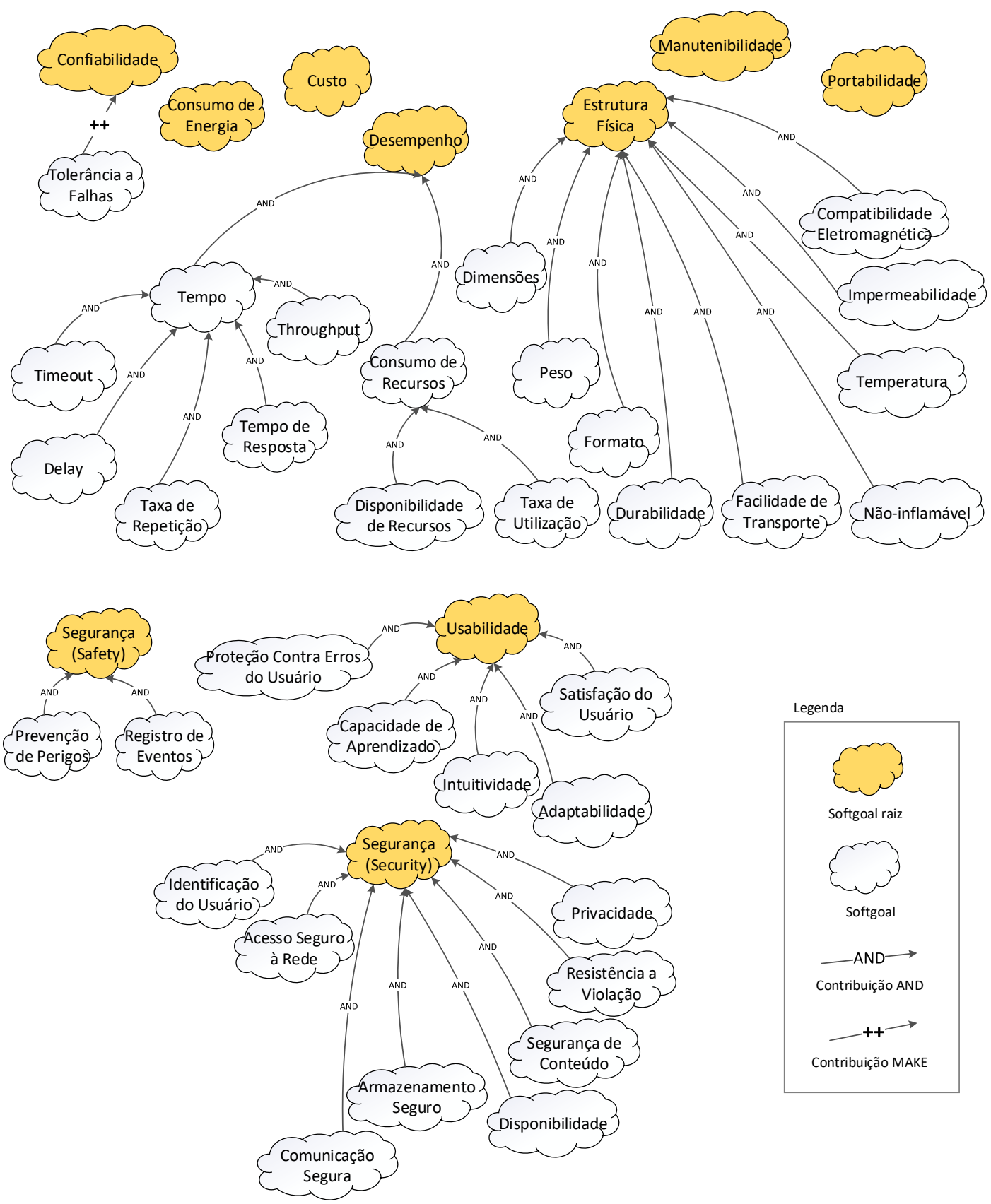

Figura 2. Grafo SIG com RNFs para Sistemas Embarcados. As correlações, omitidas para evitar poluição visual, são listadas na Tabela 1

O SIG (Figura 2) não é exaustivo nem assume a premissa de mundo fechado. Por exemplo, o fato de Desempenho ser refinado em Tempo e Consumo de Recursos não significa, necessariamente, que esses são os únicos requisitos que compõem o refinamento de Desempenho. Significa apenas que Tempo e Consumo de Recursos são dois dos possíveis refinamentos (ou dimensões) de Desempenho. 
Dado que a representação das correlações entre RNFs com o SIG é de difícil visualização, optamos por apresentar as correlações do SIG na forma de listagem (Tabela 1). Os sinais $(-,--,+,++)$ representam, respectivamente, contribuições parcialmente negativas, suficientemente negativas, parcialmente positivas e suficientemente positivas, de acordo com o NFR Framework [3].

Tabela 1. Listagem das Correlações entre os RNFs

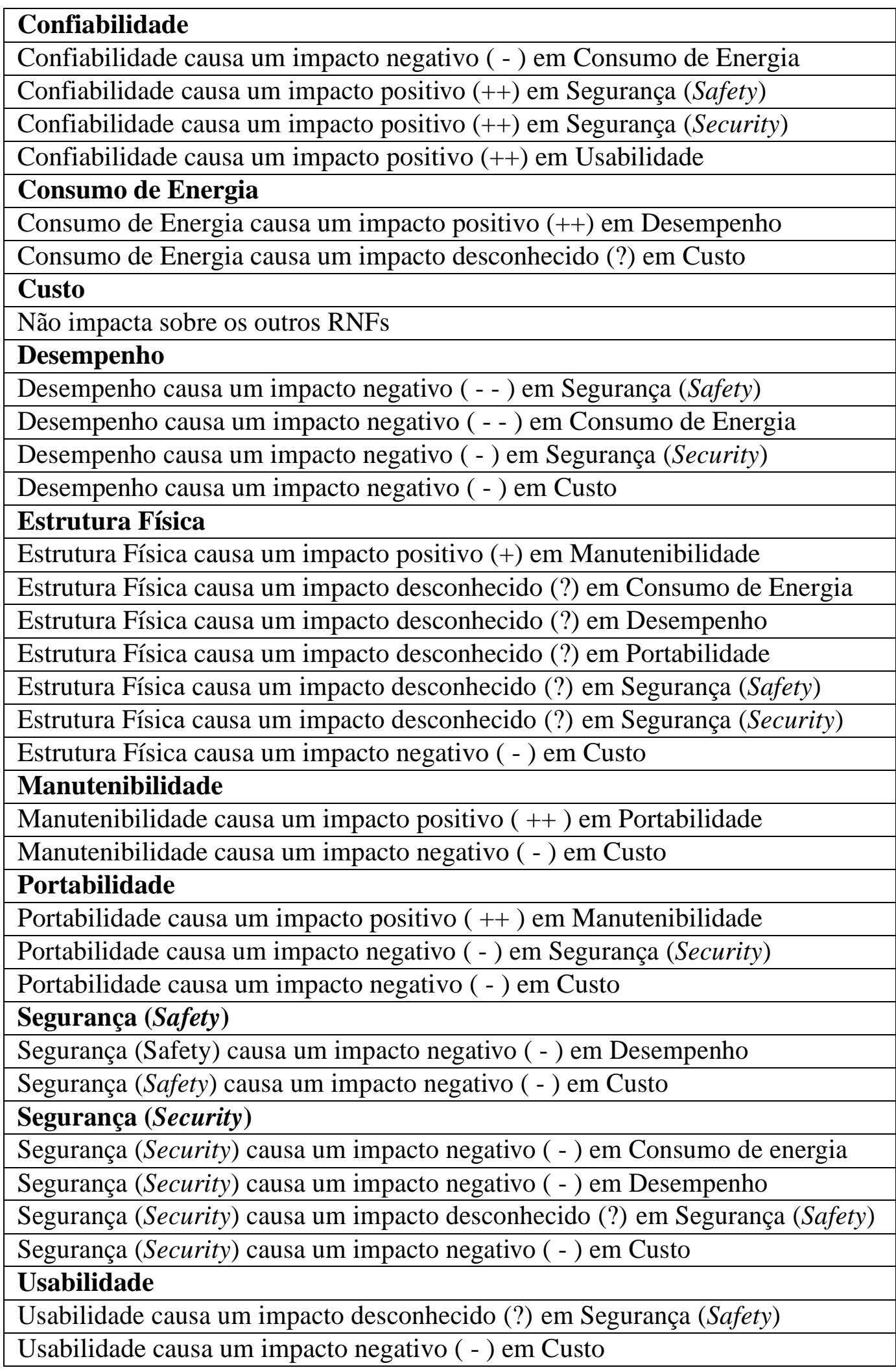


Ao contrário da identificação dos RNFs propriamente ditos, que foi baseada na literatura, as correlações apresentadas na Tabela 1 foram definidas com base em entrevistas com especialistas e refletem, portanto, o conhecimento e a experiência desses profissionais. De fato, devido à natureza subjetiva dos RNFs, e também à heterogeneidade do domínio, é possível que outros especialistas tenham opiniões divergentes. Sendo assim, essas correlações não representam uma imagem definitiva e completa da área, e sim a visão parcial dos especialistas entrevistados.

Na próxima seção, exemplificamos os demais elementos do catálogo com trechos referentes ao RNFs Confiabilidade, Consumo de Energia, Estrutura Física e Portabilidade . O catálogo completo está disponível em [6].

\subsection{Confiabilidade}

Definição: é a capacidade do sistema de fornecer serviços conforme especificado [11]. A confiabilidade do sistema também pode ser definida como a probabilidade do sistema executar sua função pretendida sob limites de projeto especificados[12]. A figura 3 expressa os refinamentos e correlações do Requisito Não-Funcional de confiabilidade.

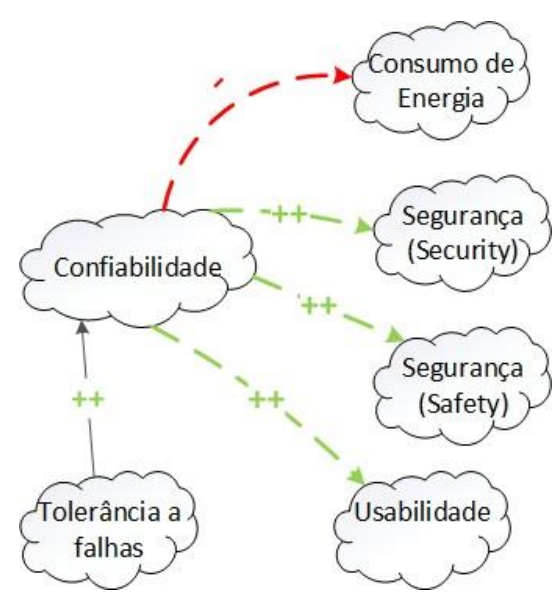

Figura 3. Requisito de Confiabilidade - Refinamentos e Correlações

Fonte: Próprio Autor

Considerar as questões relacionadas a confiabilidade desde as fases iniciais no projeto de sistemas embarcados é de fundamental importância para o sucesso do produto que será desenvolvido. Podemos citar alguns exemplos de sistemas embarcados em que a confiabilidade éum fator determinante para o seu funcionamento, tais como: sistemas de airbag, robôs autônomos, veículos aéreos não tripulados e diversas outras aplicações[13].

A figura 3 apresenta uma contribuição MAKE (++) do atributo tolerância a falhas para o requisito Confiabilidade. Isto expressa que o atributo tolerância a falhas contribui de forma suficiente positiva para confiabilidade. Além disso, são apresentadas três correlações positivas (++) para os requisitos segurança (safety), segurança (security) e usabilidade, e uma correlação negativa ( - ) para o requisito consumo de energia. Essas correlações expressam os impactos que a confiabilidade causa nestes requisitos. 
- Tolerância a falhas: visa o desenvolvimento de técnicas para evitar falhas de serviços e na presença de falhas fornecer o serviço solicitado, mesmo que em um nível degradado [14, 13]. A figura 4 apresenta um cartão de especificação que ilustra um requisito de confiabilidade que trata o atributo tolerância a falhas.

\begin{tabular}{|c|c|c|}
\hline \multicolumn{3}{|c|}{ Cartão de Especificação } \\
\hline Nr Requisito: & Classificação & Confiabilidade/Tolerância a falhas \\
\hline Descrição: & \multicolumn{2}{|c|}{$\begin{array}{l}\text { Tratamento de tolerância a falhas - implementar mecanismos de tolerância a falhas } \\
\text { através de redundância de hardware ou software }\end{array}$} \\
\hline Justificativa: & \multicolumn{2}{|c|}{$\begin{array}{l}\text { O sistema e seus dispositivos devem ser projetados para manter um estado à prova } \\
\text { de falhas, e na presença de uma condição de falha que resulte na incapacidade da } \\
\text { bomba em garantir sua integridade de operação, a bomba não deve fornecer } \\
\text { insulina e nem gerar energia ou substâncias que possam afetar a segurança do } \\
\text { usuário. }\end{array}$} \\
\hline Origem do requisito: & \multicolumn{2}{|l|}{ Projetista de hardware/software } \\
\hline Critério de aceitação: & \multicolumn{2}{|c|}{$\begin{array}{l}\text { Em caso de falha o sistema deve ser capaz de se recuperar e retornar ao seu } \\
\text { estado de pleno funcionamento em até } 5 \text { segundos. }\end{array}$} \\
\hline Dependências: & \multicolumn{2}{|l|}{ Nenhum } \\
\hline Prioridade: & \multicolumn{2}{|l|}{9} \\
\hline Conflitos: & \multicolumn{2}{|l|}{ Nenhum } \\
\hline História: & \multicolumn{2}{|l|}{$06 / 10 / 2013$} \\
\hline
\end{tabular}

Figura 4. Requisito Não-Funcional: Tratamento de tolerância a falhas

Fonte: Baseado no sistema de bomba de infusão de insulina desenvolvido por [14]

\subsection{Consumo de Energia}

Definição: são requisitos que definem estratégias para o gerenciamento e consumo de energia do sistema. É um aspecto relevante pois grande parte dos sistemas embarcados são alimentados por baterias portáteis com uma fonte limitada de energia [16]. A figura 5 expressa as correlações do Requisito Não-funcional consumo de energia.

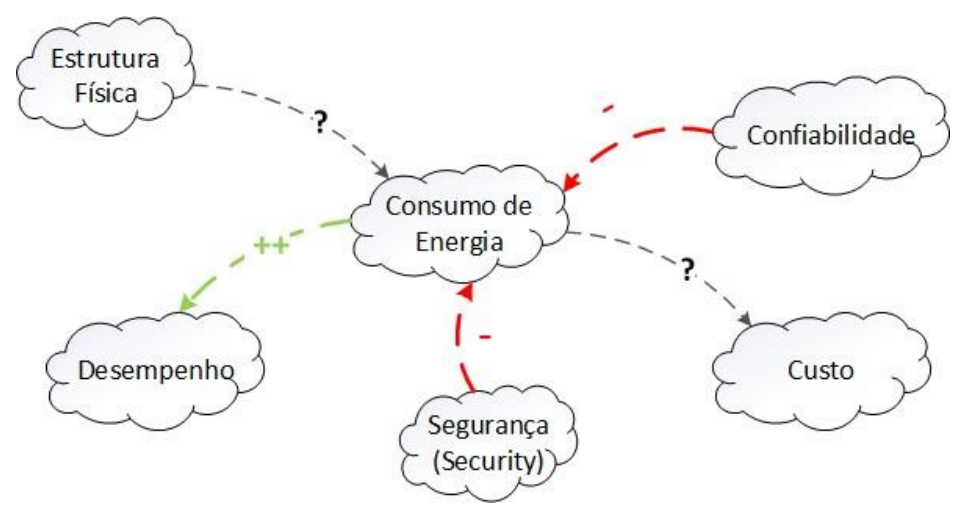

Figura 5. Requisito Consumo de Energia - Correlações

Fonte: Próprio Autor

A figura 5 apresenta uma correlação positiva(++) para o requisito Desempenho, uma correlação não identificada(?) do requisito Consumo de Energia para o requisito custo e uma correlação não identificada do requisito Estrutura Física para Consumo de 


\begin{tabular}{|l|l|l|l|}
\hline \multicolumn{2}{|c|}{ Cartão de Especificação } \\
\hline Nr Requisito: & 2 & Classificação & Consumo de Potencia/Energia \\
\hline Descrição: & $\begin{array}{l}\text { Implementar técnicas para redução do consumo de energia - o sistema deve } \\
\text { implementar técnicas para redução do consumo de energia como DVS(Dynamic } \\
\text { Voltage Scaling), DPM(Dynamic Power Management) dentre outras. }\end{array}$ \\
\hline Justificativa: & $\begin{array}{l}\text { Técnicas de redução de energia contribuem para uma melhor economia de energia } \\
\text { e maior autonomia de uso. }\end{array}$ \\
\hline Origem do requisito: & Projetista de hardware/Software \\
\hline Critério de aceitação: & $\begin{array}{l}\text { O Sistema deve possuir um consumo de 300 } \mu \mathrm{A} \text { em modo ativo e 1,1 } \mu \mathrm{A} \text { em modo } \\
\text { de espera. }\end{array}$ \\
\hline Dependências: & Nenhum \\
\hline Prioridade: & 9 \\
\hline Conflitos: & Nenhum \\
\hline História: & $08 / 10 / 2013$ \\
Figura 6. Requisito Não-funcional: Implementar técnicas para reduç ão do con- \\
sumo de energia.
\end{tabular}

Fonte: Baseado no sistema de bomba de infusão de insulina desenvolvido por [14]

Energia. Além disso, são apresentadas duas correlações negativas (-) dos requisitos Confiabilidade e Custo para o requisito Consumo de Energia. Isto implica que, consumo de energia causa um impacto positivo em desempenho. Contudo, Consumo de Energia pode causar um impacto não identificado em Custo. Estrutura Física pode causar algum impacto não identificado em Consumo de Energia. Os requisitos Confiabilidade e Segurança(security) causam um impacto negativo em Custo. A figura 6 apresenta um exemplo de um requisito relacionado ao consumo de energia.

\subsection{Estrutura f'isica}

Definição: são requisitos que expressam as características físicas sobre os componentes do sistema [17]. A figura 7 expressa os refinamentos e correlações do requisito estrutura física. Sistemas Embarcados estão fisicamente localizados em algum artefato maior, como um veículo, uma máquina de lavar, uma bomba de infusão de medicamentos e diversas outras aplicações. Dessa forma, o seu formato pode ser ditado pela estética e por restrições críticas de tamanho onde geralmente se busca o seu encaixe em pequenos espaços [18]. Observando esse cenário é fundamental definir as características físicas acerca do sistema para apoiar decisões de arquitetura [17]. 


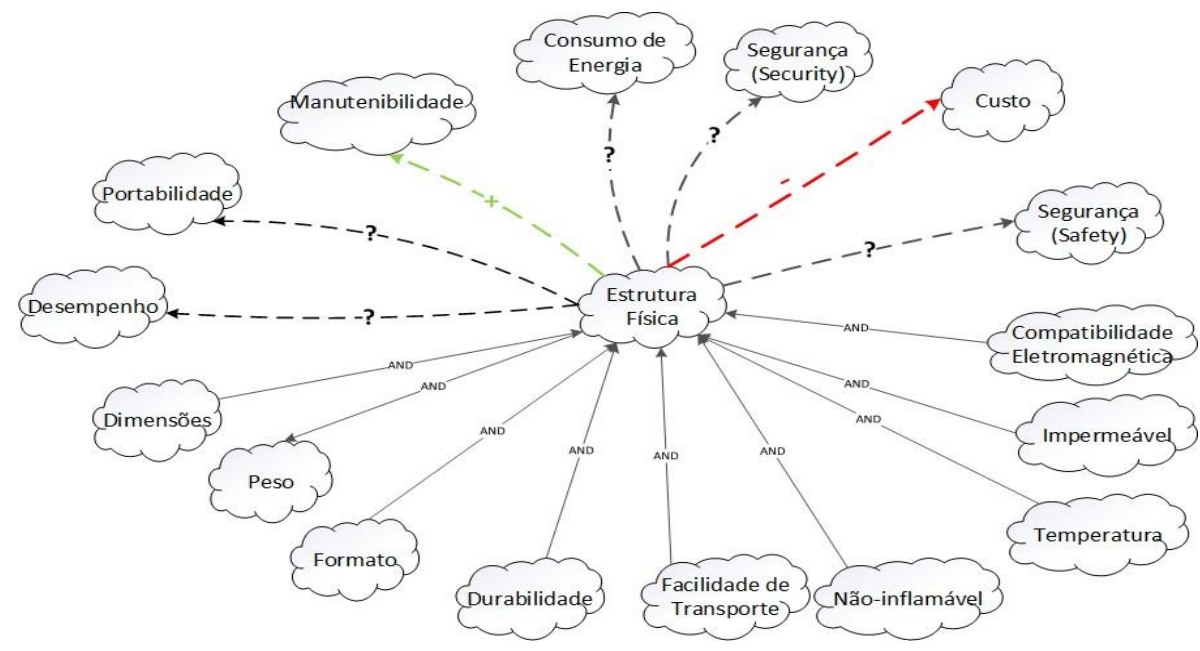

Figura 7. Requisito Estrutura Física - contribuições e correlações

Fonte: Próprio Autor

A figura 7 apresenta contribuições"AND” entre os atributos Dimensões, Peso, Formato, Durabilidade, Facilidade de Transporte, Não-Inflamável, Temperatura, Impermeável, Compatibilidade Eletromagnética para o requisito Estrutura Física. Além disso são apresentadas algumas correlações como: uma correlação parcialmente positiva (+) para o requisito Manutenibilidade, uma correlação parcialmente negativa (-) para o requisito Custo e correlações não identificadas (?) para os requisitos Desempenho, Portabilidade, consumo de Energia, Segurança (security)e Segurança (safety).

Refinamentos: Dimensões, Peso, Formato, Durabilidade, Facilidade de Transporte, Não-Inflamável, Temperatura, Impermeável e Compatibilidade Eletromagnética.

Detalhes sobre a definição de cada restrição abordada pelo RNF Estrutura física podem ser encontrados em [6], bem como um exemplo para ilustração.

\subsection{Portabilidade}

Definição: é a capacidade do sistema de ser executado sob diferentes sistemas de computação, hardware, software ou uma combinação de ambos [19]. A figura 8 expressa as correlações do requisito portabilidade.

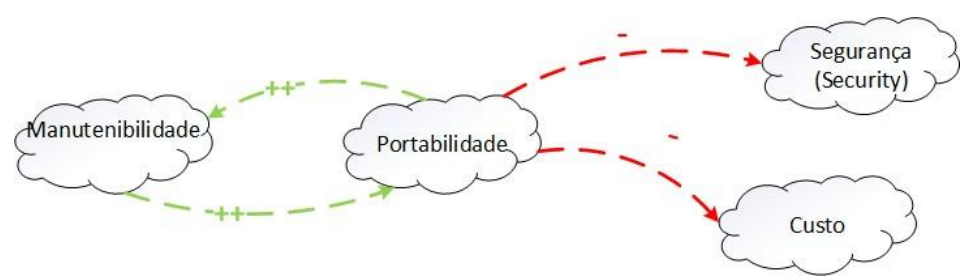

Figura 8. Requisito Portabilidade - correlações

Fonte: Próprio Autor

Em projetos de Sistemas Embarcados é comum que uma aplicação seja migrada de um sistema para outro devido a diversas necessidades que podem surgir durante o ciclo 
de vida do software. Essas necessidades vão desde a inclusão de um hardware de menor custo a recursos adicionais como memórias e componentes presentes em plataformas espec'ificas [19].

É importante ressaltar que o termo "Portabilidade" em Sistemas Embarcados muitas vezes é tratado na literatura como portável, com a ideia de algo pequeno e compacto que não é o contexto que estamos tratando neste trabalho.

A figura 8 apresenta uma correlação positiva de Portabilidade para o requisito Manutenibilidade, duas correlações parcialmente negativas para os requisitos custo e Segurança (security), e um correlação Positiva do requisito Manutenibilidade para o requisito Portabilidade. Isto implica que, a Portabilidade causa um impacto suficientemente positivo (++) em Manutenibilidade, um impacto parcialmente negativo (-) em Custo e em Segurança (security), e a Manutenibilidade causa um impacto suficientemente positivo (++) em Portabilidade.

A figura 9 apresenta um cartão de especificação para exemplificar um requisito de portabilidade.

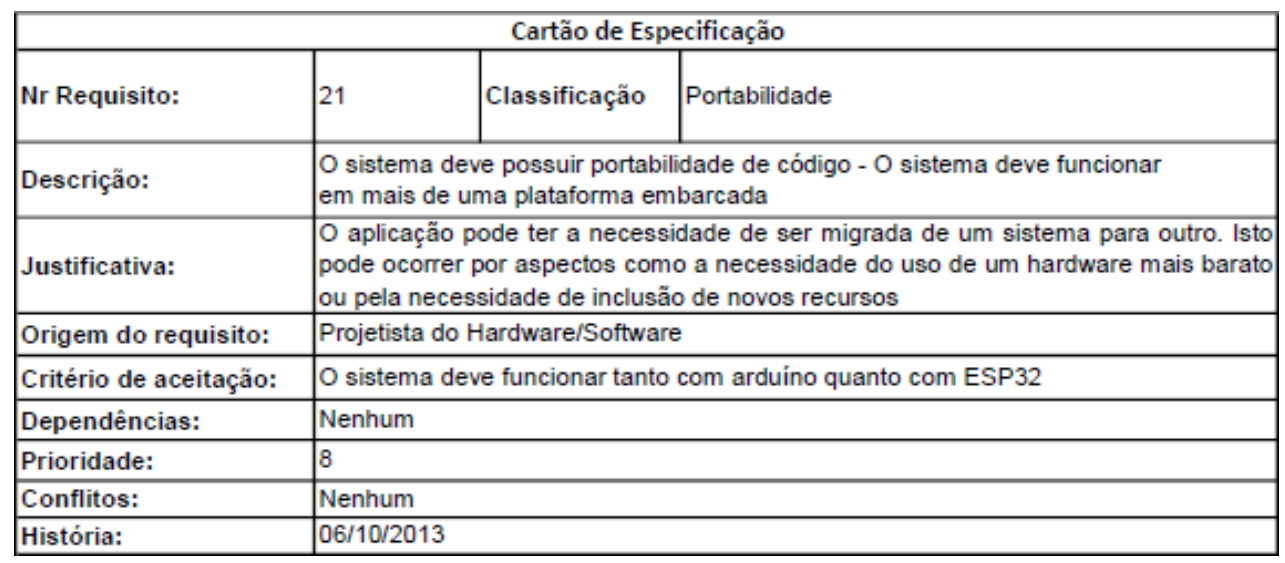

Figura 9. Requisito Não-Funcional: o sistema deve possuir portabilidade de código

Fonte: Baseado no documento de requisitos do sistema Active Park Assist 2 (Assistente de estacionamento) desenvolvido por [21]

\section{Prova de Conceito}

O sistema trabalhado nesta Prova de Conceito é o Módulo de Aquisição de Imagens para Auto-Diagnóstico (MAIA), um sistema embarcado desenvolvido por uma empresa brasileira. O MAIA consiste em um sistema eletrônico e mecânico para aquisição de imagens de microscopia no ambiente de um laboratório básico, com a finalidade de submeter essas imagens para um firmware que usa inteligência artificial para diagnosticar exames parasitológicos, urianálise e hemogramas. O principal objetivo do MAIA é a aquisição automática de imagens de microscopia em lâminas com amostras de fezes, urina ou sangue e a submissão das mesmas para um serviço na nuvem que faz a análise destas. A seguir relatamos a instanciação do catálogo NFR4ES, através do uso de 3 diretrizes, na descrição de alguns RNFs do sistema MAIA.

- $1^{\text {a }}$ Diretriz- Definição dos requisitos: Nesta etapa foi definido com base no 
catálogo quais os requisitos que são relevantes para o sistema. Para isso, foi realizada a aplicação de um questionário com o Engenheiro de Hardware da empresa. Esse questionário, disponível em [6], contemplava todos todos os RNFs presentes no catálogo NFR4ES.

- $2^{\text {a }}$ Diretriz - Instanciação do SIG: Nesta etapa, já com todos os RNFs identificados para o sistema, realizamos a instanciação do Softgoal Interdependency Graph (SIG) para o sistema sendo desenvolvido. Na Figura 10 é apresentada uma visão parcial da instanciação para o sistema MAIA, contemplando o refinamento do RNF Manutenibilidade. Neste trecho foram criados novos requisitos através de 4 refinamentos e 6 operacionalizações. A instanciação completa está disponível em [6].

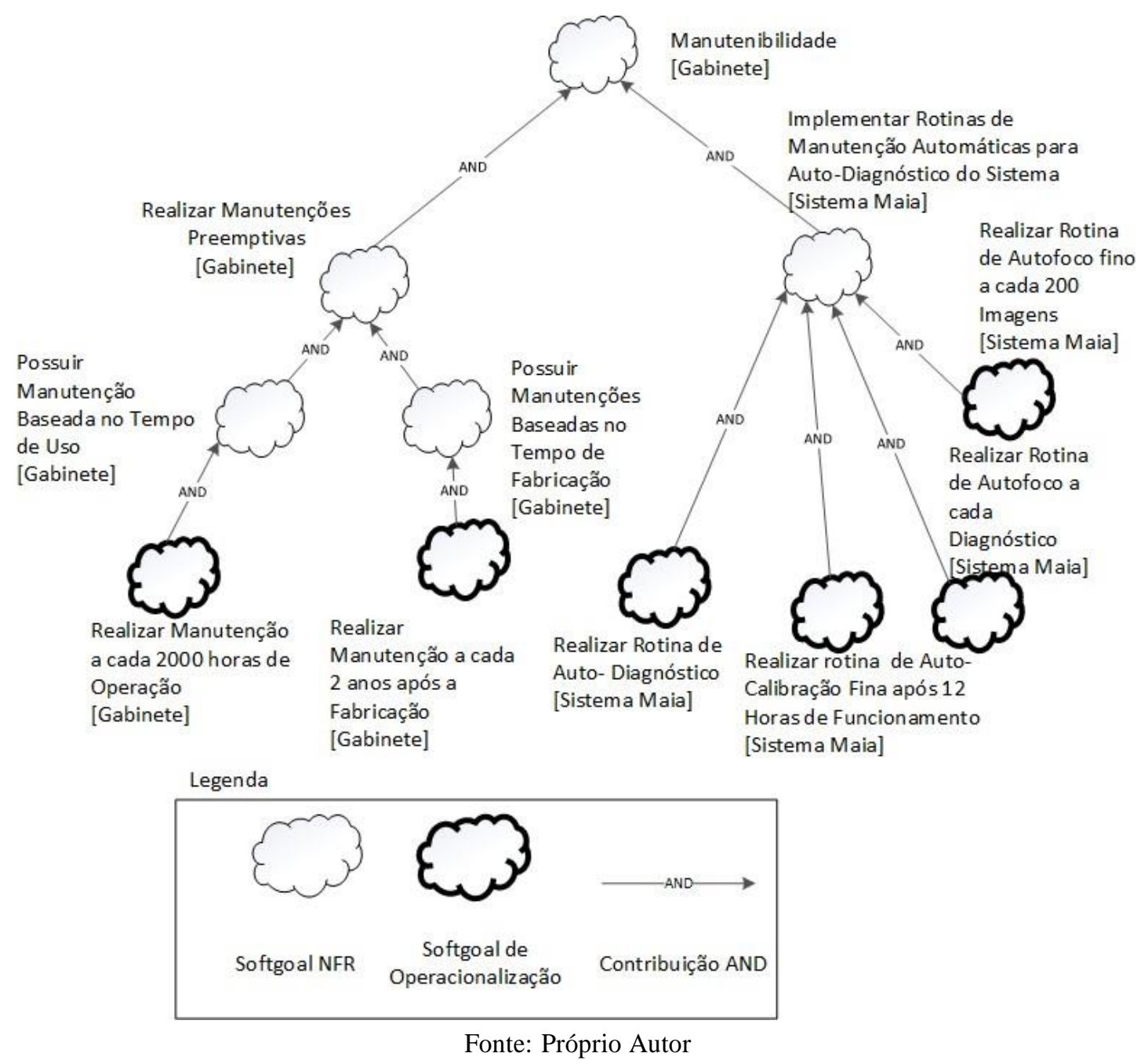

Figura 10. Refinamento do RNF Manutenibilidade para o sistema MAIA

- $3^{\text {a }}$ Diretriz - Criação dos cartões de especificação: nesta etapa foi realizada a criação dos cartões de especificação com o detalhamento dos requisitos. A Figura 11, apresenta um exemplo de RNF relacionado a temperatura do sistema.

A elicitação e especificação de requisitos do sistema MAIA já havia sido realizada previamente pelo Engenheiro de Hardware da empresa, que forneceu essas informações para que um dos autores construísse os SIGs e os cartões de 
especificação. Após a criação dos artefatos eles foram devidamente validados com o representante da empresa. Em seguida, foi administrado um questionário aberto para que pudesse ser obtido feedback sobre o catálogo. Com esta prova de conceito observamos que nossa abordagem se mostrou aplicável para a elicitação e especificação dos requisitos de sistemas embarcados.

\begin{tabular}{|c|c|c|c|}
\hline \multicolumn{4}{|c|}{ Cartão de Especificação } \\
\hline Nr Requisito: & 1 & Classificação & Estrutura Fisica/Temperatura \\
\hline Descrição: & \multicolumn{3}{|c|}{$\begin{array}{l}\text { Temperatura de funcionamento - O sistema Maia deve operar dentro de uma faixa } \\
\text { especifica de temperatura }\end{array}$} \\
\hline Justificativa: & \multicolumn{3}{|c|}{$\begin{array}{l}\text { O super aquecimento do sistema pode prejudicar o seu funcionamento e seus } \\
\text { componentes }\end{array}$} \\
\hline Origem do requisito: & \multicolumn{3}{|c|}{ Projetista de Hardware do Sistema Maia } \\
\hline Critério de aceitação: & \multicolumn{3}{|c|}{$\begin{array}{l}\text { O sistema deve operar com uma temperatura interna de funcionamento entre } 10 \mathrm{e} \\
35 \text { graus Celsius }\end{array}$} \\
\hline Dependências: & \multicolumn{3}{|l|}{ Nenhum } \\
\hline Prioridade: & \multicolumn{3}{|l|}{9} \\
\hline Conflitos: & \multicolumn{3}{|l|}{ Nenhum } \\
\hline História: & \multicolumn{3}{|l|}{$10 / 12 / 2018$} \\
\hline
\end{tabular}

Fonte: Próprio Autor

Figura 11. Requisito Não-funcional: Temperatura

\section{Avaliação por Especialistas através de Questionário}

Os participantes desta avaliação foram convidados através de contato via e-mail. Foram convidados 23 profissionais, que foram escolhidos através de um lista de contatos fornecida por um especialista do dom'inio que participou da nossa pesquisa. Dos 23 profissionais convidados, 10 realizaram a avaliação. A aplicação do questionário ocorreu tanto de forma presencial como por contato remoto (online). Na aplicação presencial, o catálogo foi disponibilizado na forma impressa e explicado para os participantes, os quais fizeram suas leituras do mesmo. Em seguida os participantes analisaram de forma detalhada o catálogo e responderam o instrumento de avaliação que considerava os aspectos abordados pelos RNFs descritos no catálogo NFR4ES. Na aplicação online, foi realizada a aplicação do instrumento de avaliação através de video-conferência, onde o catálogo foi enviado ao respondente, explicado, lido e analisado pelo respondente. O participante então recebia o questionário, respondia e o enviava de volta para um dos autores. As tabelas 2, 3 e 4 apresentam as perguntas do questionário juntamente às respostas dos participantes.

Como resultados das questões 1 a 3 concluímos que a maioria dos participantes possuem uma boa experiência para avaliarem o catálogo proposto. 


\begin{tabular}{|l|l|}
\hline \multicolumn{2}{|c|}{ Parte 1 - Identificação do perfil } \\
\hline 1. Qual sua ocupação atual & $\begin{array}{l}\text { Professor 20\% } \\
\text { Engenheiro de Requisitos 10\% } \\
\text { Desenvolvedor 70\% }\end{array}$ \\
\hline 2. Qual sua a sua maior formação? & $\begin{array}{l}\text { Graduação 40\% } \\
\text { Especialização 10\% } \\
\text { Mestrado 20\% } \\
\text { Doutorado 30\% }\end{array}$ \\
\hline $\begin{array}{l}\text { 3. Quantos anos de experiência você } \\
\text { tem no desenvolvimento de sistemas } \\
\text { embarcados? }\end{array}$ & $\begin{array}{l}10 \text { a } 20 \text { anos. 20\% } \\
\text { a } 10 \text { anos 30\% } \\
0 \text { a } 4 \text { anos 50\% }\end{array}$ \\
\hline \multicolumn{2}{|c|}{ Fonte:Próprio autor } \\
\hline
\end{tabular}

Com os resultados das questões de 4 a 10 notou-se que o catálogo NFR4ES apresenta requisitos que refletem bem a realidade de projetos de sistemas embarcados e os participantes validaram de maneira positiva a pertinência destes requisitos. As correlações definidas refletiram de maneira satisfatória os impactos de um RNF sobre outro. Porém, nos comentários das questões abertas (Tabela 4) surgiram algumas discordâncias; este é um ponto que deve ser aprimorado em trabalhos futuros. Adicionalmente, as definições presentes no catálogo se mostraram adequadas. Por último, a Tabela 4 apresenta alguns dos comentários fornecidos pelos participantes nas questões abertas.

Tabela 3. Resultados da avaliação do catálogo

\begin{tabular}{|l|l|l|l|l|l|}
\hline Perguntas/Resultados & $\begin{array}{l}\text { Discordo } \\
\text { totalmente }\end{array}$ & $\begin{array}{l}\text { Discordo } \\
\text { parcialmente }\end{array}$ & Neut. & $\begin{array}{l}\text { Concordo } \\
\text { parcialmente }\end{array}$ & $\begin{array}{l}\text { Concordo } \\
\text { totalmente }\end{array}$ \\
\hline $\begin{array}{l}\text { 4. Os Requisitos Não-funcionais } \\
\text { apresentados no catálogo NFR4ES são } \\
\text { pertinentes a projetos de Sistemas } \\
\text { Embarcados. }\end{array}$ & $70 \%$ & $30 \%$ & $0 \%$ & $0 \%$ & $0 \%$ \\
\hline $\begin{array}{l}\text { 5. As correlações apresentadas no } \\
\text { catálogo NFR4ES são condizentes } \\
\text { com a realidade de projetos de } \\
\text { Sistemas Embarcados. }\end{array}$ & $60 \%$ & $40 \%$ & $0 \%$ & $0 \%$ & $0 \%$ \\
\hline $\begin{array}{l}\text { 6. Os requisitos do catálogo NFR4ES } \\
\text { apresentam } \\
\text { definições adequadas. }\end{array}$ & $60 \%$ & $40 \%$ & $0 \%$ & $0 \%$ & $0 \%$ \\
\hline $\begin{array}{l}\text { 7. O catálogo NFR4ES é } \\
\text { de fácil entendimento. }\end{array}$ & $50 \%$ & $40 \%$ & $10 \%$ & $0 \%$ & $0 \%$ \\
\hline $\begin{array}{l}\text { 8. O catálogo NFR4ES é um artefato útil } \\
\text { nas etapas de elicitação e especificação } \\
\text { no processo de Engenharia } \\
\text { de Requisitos para Sistemas } \\
\text { Embarcados. }\end{array}$ & $60 \%$ & $40 \%$ & $0 \%$ & $0 \%$ & $0 \%$ \\
\hline $\begin{array}{l}\text { 9.Eu utilizaria o catálogo } \\
\text { NFR4ES em projetos futuros. }\end{array}$ & $80 \%$ & $20 \%$ & $0 \%$ & $0 \%$ & $0 \%$ \\
\hline $\begin{array}{l}\text { 10. Eu recomendaria a outras pessoas a } \\
\text { utilização do } \\
\text { catálogo NFR4ES. }\end{array}$ & $60 \%$ & $40 \%$ & $0 \%$ & $0 \%$ & $0 \%$ \\
\hline
\end{tabular}


Tabela 4. Sugestões para o catálogo

\begin{tabular}{|c|}
\hline Parte 3 - Finalização (sugestões) \\
\hline $\begin{array}{l}\text { 11. Há algum requisito no projeto de sistemas embarcados que não foi citado e você } \\
\text { gostaria de destacar e acrescentar no catálogo? }\end{array}$ \\
\hline $\begin{array}{l}\text { Observação 1:Um dos participantes apontou, como requisitos que podem ser acrescentados no } \\
\text { catálogo, os requisitos ligados a comunicação e mencionou que eles são fundamentais } \\
\text { em Internet of Things (IOT). Porém, não mencionou nenhum destes requisitos. }\end{array}$ \\
\hline $\begin{array}{l}\text { Observação 2: um participante fez uma observação que o requisito time-to-market pode } \\
\text { estar associado a custo ou a tempo }\end{array}$ \\
\hline $\begin{array}{l}\text { Observação3: um participante citou que um requisitos de Modularização deveria } \\
\text { ser adicionado }\end{array}$ \\
\hline 12. Há alguma correlação que você gostaria de modificar ou acrescentar no catálogo? \\
\hline $\begin{array}{l}\text { Observação 1: Um dos participantes fez a observação que os Custos do Produto derivam } \\
\text { dos custos do projeto, e isso impacta em outros RNFs. }\end{array}$ \\
\hline $\begin{array}{l}\text { Observação 2: um participante teve a opinião que Consumo de Energia causa um impacto } \\
\text { positivo em Custo (maior consumo, maior custo) }\end{array}$ \\
\hline 13. Existem outras modificações que podem ser feitas para melhorar o catálogo? Quais? \\
\hline $\begin{array}{l}\text { Observação 1: um participante fez a observação que a exibição dos Requisitos em nuvens } \\
\text { com setas representando correlações fica um pouco confusa e a primeira vista pode } \\
\text { assustar o usuário do catálogo }\end{array}$ \\
\hline $\begin{array}{l}\text { Observação 2; um participante disse que trocaria Satisfação do Usuário por Experiência do } \\
\text { Usuário ou manteria as duas. Ele questionou se Facilidade de Transporte não entraria } \\
\text { em portabilidade, e faria a inclusão do requisito modularização, que seria a possibilidade de } \\
\text { utilizá-lo com outros módulos }\end{array}$ \\
\hline
\end{tabular}

Fonte:Próprio autor

\section{Trabalhos Relacionados}

A Tabela 5 apresenta quatro trabalhos que tem relação com a nossa pesquisa. Os critérios de comparação foram definidos de acordo com as principais características do catálogo: a utilização de grafos do NFR Framework, tratamento especifico de RNFs, apoio às atividades de elicitação e especificação e a construção de um catálogo genérico de RNFs para sistemas embarcados.

O trabalho realizado por Pereira et al. [9] apresenta uma investigação sobre a especificação de RNFs no domínio de sistemas embarcados. Os trabalhos desenvolvidos por Ossada et al. [22] e Martins et al. [23] apresentam abordagens de Engenharia de Requisitos para apoiar projetos de sistemas embarcados que também visam apoiar as atividades de elicitação e especificação de requisitos. Contudo, eles não fornecem a catalogação de RNFs. O trabalho desenvolvido por Toniolo e Martins [24], além de apresentar uma catalogação com o NFR Framework, pode ser utilizado para elicitar e especificar famílias de produtos. Em nosso trabalho a proposta é utilizar catálogos do NFR Framework para apoiar projetos de sistemas embarcados, onde esses catálogos podem ser aplicados em diversos sub-domínios. Para uma análise mais detalhada dos trabalhos relacionados consultar [8]. 
Tabela 5. Comparação dos trabalhos relacionados sobre aspectos observados

\begin{tabular}{|l|c|c|c|c|}
\hline $\begin{array}{l}\text { Aspectos observados } \\
\text { Trabalhos relacionados }\end{array}$ & $\begin{array}{c}\text { Apresentam } \\
\text { catálogos com o } \\
\text { NFR framework }\end{array}$ & $\begin{array}{l}\text { Tipos de } \\
\text { Requisitos }\end{array}$ & Atividades apoiadas & $\begin{array}{l}\text { Apresenta } \\
\text { um catalogo } \\
\text { genérico } \\
\text { para SEs }\end{array}$ \\
\hline (Cavalcante,2017) & Não & RNF & Especificação & Não \\
\hline (Ossada et al.,2012) & Não & RF e RNF & Elicitação e Especificação & Não \\
\hline (Martins et al.,2010) & Não & RNF & Especificação & Não \\
\hline (Toniolo el a,,2018) & Sim & RNF & Elicitação e especificação & Sim \\
\hline NFR4ES & Sim & RNF & Elicitação e Especificação & Sim \\
\hline
\end{tabular}

\section{Conclusões e Trabalhos Futuros}

Este artigo tratou da dificuldade de identificar, inter-relacionar e documentar RNFs em projetos de sistemas embarcados. A questão de pesquisa 1 (Quais os principais RNFs que devem ser levados em consideração em projetos de sistemas embarcados?) foi respondida através da coleta de evidências na literatura acadêmica da aplicação dos RNFs catalogados em projetos de sistemas embarcados. A questão 2 (Quais são os interrelacionamentos entre os principais RNFs para sistemas embarcados?) foi respondida através da documentação das correlações entre os RNFs catalogados com base na opinião de quatro especialistas na área. O catálogo foi aplicado com sucesso em uma prova de conceito, além de ter sido avaliado por outros dez especialistas. A partir dos resultados obtidos nesta pesquisa e as limitações encontradas, são propostas algumas ações como trabalhos futuros: aperfeiçoar o catálogo com novos RNFs de produto baseados em padrões da indústria, normas técnicas e a opinião de mais desenvolvedores de sistemas embarcados; expandir o catálogo de RNFs, com a inclusão de requisitos de processo e requisitos externos; e aplicar o catálogo no desenvolvimento de novos sistemas embarcados.

\section{Agradecimentos}

Os autores agradecem ao apoio financeiro do Conselho Nacional de Desenvolvimento Científico e Tecnológico (CNPq) e a da Fundação de Amparo à Ciência e Tecnologia do Estado de Pernambuco (FACEPE).

\section{Referências}

[1] Jaime Cazuhiro Ossada and Luiz Eduardo Galvão Martins. Um estudo de campo sobre o estado da prática da elicitação de requisitos em sistemas embarcados. In Workshop em Engenharia de Requisitos (WER), Cuenca, Equador, Abril 12-13, 2010, pages 41-52, 2010.

[2] Aêda Sousa, Celso Agra, Josenildo Melo, and Fernanda MR Alencar. Elicitação e especificação de requisitos em sistemas embarcados: Uma revisão sistemática. In Workshop de Engenharia de Requisitos (WER), 2015.

[3] Lawrence Chung, Brian A Nixon, Eric Yu, and John Mylopoulos. Non-functional requirements in software engineering. 2000.

[4] Dewi Mairiza, Didar Zowghi, and Nurie Nurmuliani. An investigation into the notion of non-functional requirements. In Proceedings of the 2010 ACM Symposium on Applied Computing, pages 311-317, ACM, 2010. 
[5] Milton Deivson Albuquerque CAVALCANTE. Uma análise sobre a engenharia de requisitos em sistemas embarcados. (Mestrado Profissional) - Programa de PósGraduação em Ciência da Computação - Universidade Federal de Pernambuco, 3 2017.

[6] Reinaldo Silva. Nfr4es: Um catálogo de requisitos não-funcionais para sistemas embarcados. (Mestrado). Programa de Pós-Graduação em Ciência da Computação Universidade Federal de Pernambuco (UFPE), 2019.

[7] Reinaldo Silva, Jaelson Castro, João Pimentel. Nfr4es: Um catálogo de requisitos nãofuncionais para sistemas embarcados. Anais do WER20 - Workshop em Engenharia de Requisitos, São José dos Campos, SP, Brasil, Agosto 24-28, 2020, 2020.

[8] Business Process Modeling Notation OMG. Version 1.0. OMG Final Adopted Specification, Object Management Group, 190, 2006.

[9] Tarcísio Pereira, Deivson Albuquerque, Aêda Sousa, Fernanda MR Alencar, and Jaelson Castro. Retrospective and trends in requirements engineering for embedded sys- tems: A systematic literature review. In Conferencia Iberoamericana de Software Engineering 2017 (CIbSE), pages 427-440, 2017.

[10]Suzanne Robertson and James Robertson. Mastering the requirements process: Getting requirements right. Addison-wesley, 2012.

[11]Jin-Hui Shan, Hai-Yan Zhao, Jin-Bo Wang, Rui-Xue Wang, Cheng-Lin Ruan, and Zhe$\mathrm{Xi}$ Yao. An extended tasm-based requirements modeling approach for real-time embedded software: An industrial case study. In Software Engineering and Methodology for Emerging Domains, pages 19-34. Springer, 2016.

[12]Chin-Yu Huang and Yung-Ruei Chang. An improved decomposition scheme for assessing the reliability of embedded systems by using dynamic fault trees. Reliability Engineering \& System Safety, 92(10):1403-1412, 2007.

[13]Hananeh Aliee. Reliability analysis and optimization of embedded systems using stochastic logic and importance measures. 2017.

[14]Algirdas Avizienis, J-C Laprie, Brian Randell, and Carl Landwehr. Basic concepts and taxonomy of dependable and secure computing. IEEE transactions on dependable and secure computing, 1(1):11-33, 2004.

[15]Luiz Eduardo G Martins, Hanniere de Faria, Lucas Vecchete, Tatiana Cunha, Tiago de Oliveira, Dulce E Casarini, and Juliana Almada Colucci. Development of a lowcost insulin infusion pump: Lessons learned from an industry case. In Computer-Based Medical Systems (CBMS), 2015 IEEE 28th International Symposium on, pa- ges 338343. IEEE, 2015.

[16]A.NANDINI V.DHIVYA. Estimation of energy consumption in embedded systems. In Proceedings of 5th IRF IRF International Conference, Chennai, 23rd March. 2014, pages 22-23. Digital Explore, 2014.

[17]Marilyn Wolf. Computers as components: principles of embedded computing system design. Elsevier, 2012.

[18]Adrian Boyer. What is systems engineering for embedded systems? In INCOSE Interna- tional Symposium, volume 18, pages 2071-2087, Wiley Online Library, 2008. 
[19]Mari Matinlassi. Evaluating the portability and maintainability of software product family architecture: Terminal software case study. In Software Architecture, 2004.

WICSA 2004. Proceedings. Fourth Working IEEE/IFIP Conference on, pages 295298, IEEE, 2004.

[20]Hugo Marcondes, Arliones Stevert Hoeller Junior, Lucas Francisco Wanner, and Antônio Augusto M Fröhlich. Portabilidade de sistemas operacionais no domínio de sis- temas embarcados. Relatório Técnico. Universidade Federal de Santa Catarina. Laboratório de Integração de Software e Hardware, 2004.

[21]Joseph Reeder, Ethan Ettema, Ryan Boyce, Kenneth Massie, and Stephanie Brown. Pro- ject active park assist 2. Dispon'1vel em https://bit.ly/2WceEJB, 2014.

[22]Jaime Cazuhiro Ossada, Luiz Eduardo Galvão Martins, Bárbara Stefani Ranieri, and An- derson Belgamo. Gerse: Guia de elicitação de requisitos para sistemas embarcados. In Workshop de Engenharia de Requisitos (WER), 2012.

[23]Luiz Eduardo Galvão Martins, Roberto de Souza Júnior, Hermano Peixoto de Oliveira Jr, and Cecilia Sosa Arias Peixoto. Terase: Template para especificação de requisitos de ambiente em sistemas embarcados. In Workshop de Engenharia de Requisitos (WER), 2010.

[24] Cristiano Marçal Toniolo and Luiz Eduardo Galvão Martins. PRReSE - Process of Non-Functional Requirements Reuse for Embedded Systems Based on a NFRFramework. In ICSEA 2014 : The Ninth International Conference on Software Engineering Advances, pages 293-299, IARIA, 2014 


\section{APÊNDICE A - Evidências da aplicabilidade dos RNFs a sistemas embarcados. $O$ prefixo $S$ indica que a fonte é oriunda da RSL [8], enquanto $F$ indica que é uma fonte adicional. A listagem dessas referências encontra-se em [6]}

Confiabilidade: S16, S40, S56, S65, S68, S70, S81 e F29

Tolerância a falhas: F1, F29, F32 e F36

Consumo de Energia: S07, S13, S38, S43, S47, F14 e F19

Custo: S03, S13 e S25

Desempenho: S02, S03, S13, S17, S18, S24, S38, S43, S46, S47, S56, S65, S68, S75 e S76

Tempo: S01, S02, S13, S16, S20, S24, S31, S33, S34, S39, S46, S52, S56, S63, S75, S84 e F29

Timeout: S24, F35 e F37

Throughput: S24, F31, F32, F33, F34 e F35

Delay: S63, F31, F32, F33, F34 e F35

Taxa de Repetição: S63 e F38

Tempo de Resposta: S24, F38, F39, F40 e F41

Consumo de Recurso: S24, S31, S39, F29 e F42

Disponibilidade de Recursos: S24

Taxa de Utilização: S24

Estrutura f' 1sica: S13, F4, F5, F6, F13, F14 e F22

Dimensões: S13, F5 e F22

Peso: F4, F5 e F22

Formato: F5

Durabilidade: F5

Facilidade de transporte: F6

Não-inflamável: F5

Impermeabilidade: F7 e F49

Compatibilidade eletromagnética: F13, F48 e F49

Manutenibilidade: S16, S81, F15, F16, F17, F18, F29 e F30

Portabilidade: S16, F19, F20, F21, F22, F23 e F29

Segurança (Safety): S03, S05, S06, S07, S08, S12, S14, S19, S25,

S27, S33, S35, S40, S41, S43,S45, S46, S49, S50, S51, S56, S61,

S64, S70, S74, S76, S81, S82 e F1

Prevenção de Perigos:F12

Registro de Eventos:F12

Segurança (Security): S16, S40, S48, S64, S70 e S81

Identificação do Usuário: F11, F44 e F47

Acesso Seguro a Rede: F11, F44 e F45

Comunicação Segura: F11, F44 e F45

Armazenamento Seguro: F11, F43 e F44

Disponibilidade: S38, S70, S81, F11, F44 e F46

Segurança de Conteúdo: F11 e F44

Resistência a Violação: F11, F13, F43 e F44

Privacidade: S81, F24, F25, F26 e F27

Usabilidade: S16, F5, F8, F9, F10 e F28

Capacidade de aprendizado: F8 e F10

Proteção contra erros dos usuário: F8

Adaptabilidade: F9

Satisfação do Usuário: F10

Intuitividade: F10 